\title{
ТРАНСФОРМАЦИЯ РОЛИ МАРКЕТИНГОВЫХ КАНАЛОВ В УСЛОВИЯХ ЦИФРОВИЗАЦИИ
}

\author{
(c) 2021 Дудаков Георгий Сергеевич \\ магистрант, Кафедра экономики исследований и разработок, Экономический факультет \\ Санкт-Петербургский государственный университет, Россия, Санкт-Петербург \\ E-mail: dudakov.gs@mail.ru
}

\section{(c) 2021 Молчанов Николай Николаевич}

доктор экономических наук, профессор,

заведующий Кафедрой экономики исследований и разработок, Экономический Факультет

Санкт-Петербургский государственный университет, Россия, Санкт-Петербург

E-mail: n.molchanov@spbu.ru

За последние несколько лет b2c маркетинг значительно изменился. Отмечается рост использования цифровых технологий, увеличение общего количества производимого компаниями контента, и, как следствие, изменение потребительской модели поведения с точки зрения применения цифровой информации, что обуславливает актуальность темы исследования. В сложившихся условиях клиенты становятся более избирательны, их требования к компаниям значительно повышаются. Потребители сами определяют и заявляют, чем их могут привлечь потенциальные поставщики. Крайне важным становится использование цифровых маркетинговых каналов, которые являются важнейшей составляющей частью цифрового маркетинга.

Ключевые слова: цифровой маркетинг, маркетинг, цифровые маркетинговые каналы, потребители.

Ускоренное развитие технологий обусловило трансформацию существующей экономической модели. Экономические отношения вступили на новый этап развития - цифровую эру, главной характеристикой которой является использование цифровых технологий при осуществлении хозяйственной деятельности. В свою очередь, данные изменения обусловили и трансформацию всей маркетинговой деятельности, перехода к применению инструментариев цифрового маркетинга и, как следствие, трансформацию маркетинговых каналов от традиционных к цифровым.

Цифровой маркетинг представляет собой продвижение товаров и услуг с применением цифровых технологий, в том числе цифровых маркетинговых каналов [13]. Согласно мнению Д.А.Шевченко ««цифровой маркетинг» глубже определения «интернет-маркетинг», поскольку включает в себя каналы, которые не предполагают использования сети интернет». Определения цифрового маркетингового канала представлены в табл. 1.

Таким образом, цифровой маркетинговый канал представляет собой маршрут движения информации относительно товаров и услуг, выступающий в роли коммуникатора между ком- паниями и потребителями. Основная функция цифровых маркетинговых каналов - обмен информацией и организация связи между сторонами. В качестве сторон могут быть представлены потребитель и рекламопроизводитель.

Рекламопроизводитель с помощью инструментов продвижения обеспечивает потребителя необходимой информацией о товарах (бренд, материал изделий, производитель, краткая характеристика товара и т.д.), а потребитель, основываясь на полученной информации анализирует все возможные альтернативы выбора и осуществляет покупку. Более подробно рассмотрим и сравним традиционные и цифровые маркетинговые каналы в табл. 2.

Ключевой целью как офлайн, так и онлайнмаркетинговых каналов является донесение информации до потребителей о товарах и услугах компании, повышение спроса и их заинтересованности. Что касается инструментов цифровых маркетинговых каналов, то к таковым относят мероприятия, которые дают возможность донести определенного рода информацию до требующегося количества людей или же сфокусироваться и привлечь внимание целевой аудитории.

Необходимо отметить, что отличительной чертой функционирования предприятий в циф- 
Таблица1. Трактовка понятия цифрового маркетингового канала [Составлено авторами]

\begin{tabular}{|c|c|c|}
\hline Определение & Автор определения & Трактовка понятия \\
\hline \multirow[t]{3}{*}{ Цифровой маркетинг } & $\begin{array}{l}\text { Т.П. Данько } \\
\text { О.В.КИтова [6] }\end{array}$ & $\begin{array}{l}\text { Цифровой маркетинг - это маркетинг, обеспечивающий } \\
\text { взаимодействие с клиентами и бизнес-партнёрами с исполь- } \\
\text { зованием цифровых информационно-коммуникационных } \\
\text { технологий и электронных устройств с целью формирования } \\
\text { сетей взаимодействия. }\end{array}$ \\
\hline & $\begin{array}{l}\text { Coviello, Milley \& } \\
\text { Marcolin [11] }\end{array}$ & $\begin{array}{l}\text { Цифровой маркетинг - это использование возможностей } \\
\text { интернета и других интерактивных технологий для создания } \\
\text { и налаживания диалога между компаниями и потребителями. }\end{array}$ \\
\hline & Д.А. Шевченко [1] & $\begin{array}{l}\text { Цифровой маркетинг - это маркетинг продвижения продук- } \\
\text { тов и услуг с использованием цифровых каналов для охвата } \\
\text { потребителей. }\end{array}$ \\
\hline \multirow[t]{2}{*}{$\begin{array}{l}\text { Цифровой маркетин- } \\
\text { говый канал }\end{array}$} & С. Холл [3] & $\begin{array}{l}\text { Цифровой маркетинговый канал - транспортный маршрут, в } \\
\text { котором движение - это контент, который этот канал предо- } \\
\text { ставляет. }\end{array}$ \\
\hline & Д.А. Шевченко & $\begin{array}{l}\text { Цифровой маркетинговый канал - материально-техническое } \\
\text { пространство, которое предназначено для организации связи } \\
\text { между сторонами, передачи и обмена информацией. }\end{array}$ \\
\hline
\end{tabular}

Таблица 2. Каналы и инструменты цифрового и офлайн-маркетинга [2]

\begin{tabular}{|c|c|c|c|}
\hline $\begin{array}{c}\text { Офлайн маркетинговые } \\
\text { каналы }\end{array}$ & $\begin{array}{c}\text { Инструменты офлайн- } \\
\text { маркетинга }\end{array}$ & $\begin{array}{c}\text { Цифровые маркетинго- } \\
\text { вые каналы }\end{array}$ & $\begin{array}{c}\text { Инструменты цифрового } \\
\text { маркетинга }\end{array}$ \\
\hline Прямые продажи & Журналы, брошюры & Сайты и блоги & Баннерная реклама \\
\hline Наружная реклама & Билборды, постеры & Социальные сети & Таргетированная реклама \\
\hline Рекламные ролики в СМИ & Телевидение & ПК или планшеты & Новые медиа (медиа+) \\
\hline $\begin{array}{l}\text { Тематические меропри- } \\
\text { ятия }\end{array}$ & $\begin{array}{l}\text { Письменные приглаше- } \\
\text { ния }\end{array}$ & $\begin{array}{l}\text { Вебинары, Электронная } \\
\text { почта }\end{array}$ & Email-рассылка \\
\hline Связь с общественностью & $\begin{array}{l}\text { Телевидение, журналы } \\
\text { или газеты }\end{array}$ & Смартфоны & Контекстная реклама \\
\hline
\end{tabular}

ровой среде является высокая информированность и осведомленность потребителей относительно приобретаемых ими товаров, доступ к большим объемам информации, возможность совершения покупки в любой удобный момент времени, что повышает требования к формированию маркетинговой стратегии и выстраиванию цифровых маркетинговых каналов. Далее, рассмотрим цифровые маркетинговые каналы на конкретных примерах.

Одними из наиболее популярных цифровых маркетинговых каналов являются смартфоны. Согласно данным аналитического агентства Media Scope, ежемесячная аудитория людей, которые используют смартфоны для выхода в интернет, составляла $71 \%$ от их общего числа в 2020 году [12]. Среднее время препровождения в интернете через смартфон в зависимости от возрастной группы представлено на рис. 1.

Согласно данным, представленным на рис. 1 , вне зависимости от возрастной категории, для выхода в интернет, пользователи отдают предпочтение смартфонам. Длительность проведенного ими времени в сети с помощью смартфона в среднем в 2021 году больше чем в два раза практически во всех возрастным категориях, за исключением категории пользователей старше 55 лет.

Если в 2003 году смартфоны выступали в качестве товара, доступного определенной узкоспециализированной целевой аудитории (так называемые «предметы роскоши»), которые применялись предпочтительно руководителями высшего звена, то сегодня смартфон может позволить иметь себе практически каждый, что указано на рис. 2 .

Таким образом, количество пользователей смартфонами в период с 2015 по 2020 гг. увеличилось на 55 млн. человек (на 107\%). Важно также отметить, что смартфоны на данный момент 


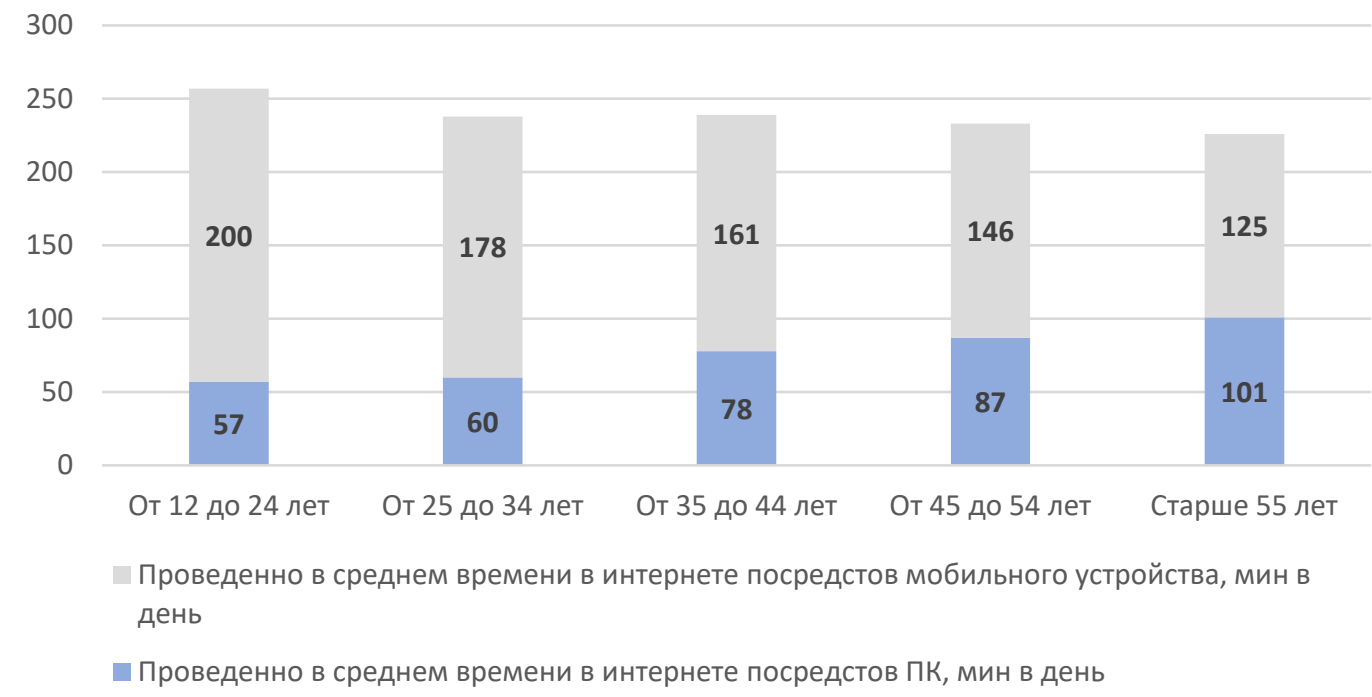

Puc. 1. Количество времени, проведенного пользователями в сети интернет через ПК и смартфон в 2021 г. в среднем [Составлено авторами на основании материалов 7]

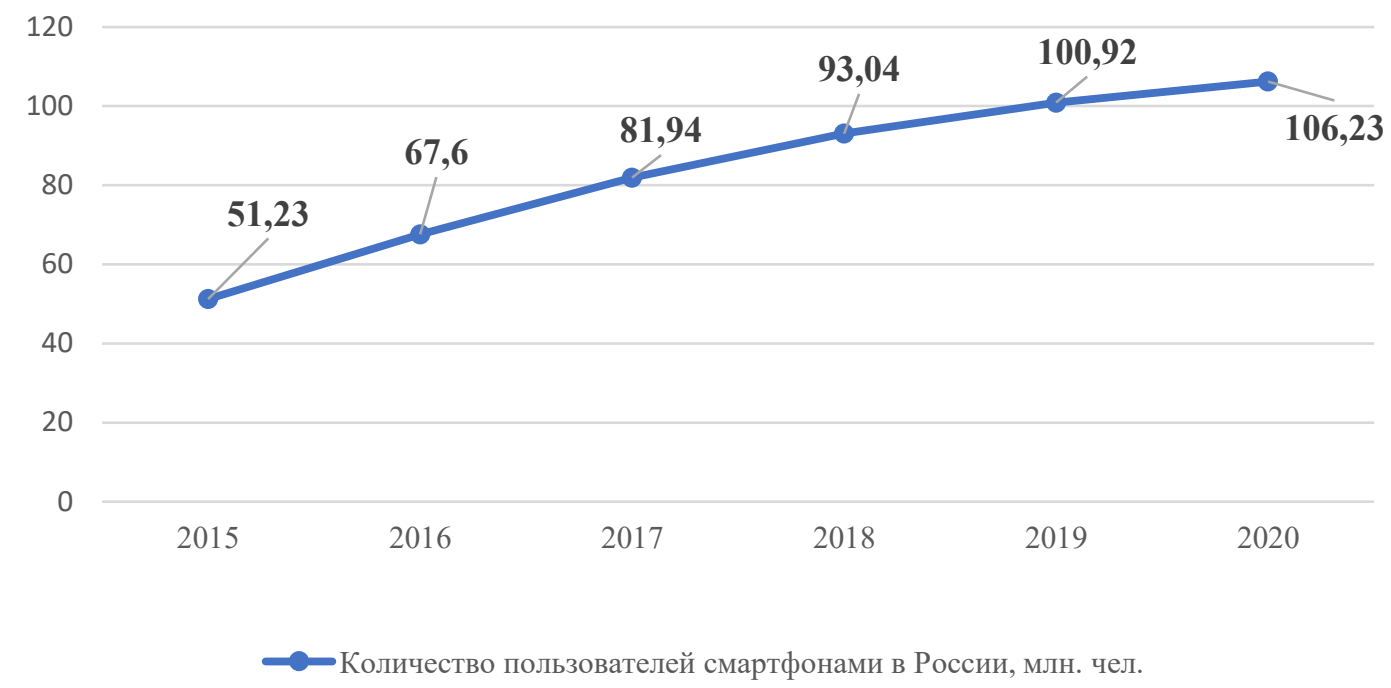

Рuc. 2. Количество пользователей смартфонами в России с 2015 по 2020 гг., млн. чел. [9]

являются более мощными не только в плане производительности, но и обмена информацией. За счет указанных изменений компании стали предоставлять большее количество информации и контента через мобильные устройства, что значительно повысило актуальность применения данных цифровых маркетинговых каналов [14].

Следующими не менее востребованными цифровыми маркетинговыми каналами являются социальные сети. Социальные сети представляют собой интернет-площадки, сайты, которые позволяют зарегистрированным на них пользователям размещать информацию о себе и коммуницировать между собой, устанавливая социальные связи [5]. В табл. 3 представлены наиболее востребованные социальные сети в Российской Федерации в 2020 году.

Согласно данным, приведенным в табл. 3, наиболее популярными социальными сетями по средней продолжительности пребывания являются «TikTok» (33 мин в день), «Вконтакте» (32 мин. в день) и «Instagram» (28 мин. в день).

Необходимо отметить, что повышение присутствия пользователей в социальных сетях фиксируются и коррелируются поисковыми системами «Яндекс», «Google» и т.п. Поисковая 
Таблица 3. Наиболее востребованные социальные сети в Российской Федерации в 2020 году [10]

\begin{tabular}{|c|c|c|c|c|}
\hline $\begin{array}{l}\text { Наименование } \\
\text { социальной сети }\end{array}$ & $\begin{array}{c}\text { Основная возрастная } \\
\text { группа пользователей }\end{array}$ & $\begin{array}{c}\text { Средняя продолжи- } \\
\text { тельность пребыва- } \\
\text { ния на платформе } \\
\text { в день }\end{array}$ & $\begin{array}{c}\text { Сообщений (лю- } \\
\text { бых открытых или } \\
\text { публичных сооб- } \\
\text { щений, включая } \\
\text { комментарии) от- } \\
\text { правлено в среднем } \\
\text { в мес. }\end{array}$ & $\begin{array}{c}\text { Количество авторов } \\
\text { (пользователей, } \\
\text { которые написали } \\
\text { хотя бы } 1 \text { публичное } \\
\text { сообщение) в месяц }\end{array}$ \\
\hline TikTok & До 34 лет & 33 мин. & 29 млрд. & 5,3 млн. \\
\hline Вконтакте & До 44 лет & 32 мин. & 496,2 млрд. & 28,7 млн. \\
\hline Instagram & $\begin{array}{l}\text { От } 12 \text { до } 44 \text { лет (48\% } \\
\text { пользователей до } 35 \\
\text { лет) }\end{array}$ & 28 мин. & 256,2 млрд. & 42,8 млн. \\
\hline Одноклассники & $\begin{array}{l}\text { Старше } 25 \text { лет } \\
\text { (42\% пользователей } \\
\text { от } 45 \text { лет) }\end{array}$ & 17 мин. & 108,6 млрд. & 6,6 млн. \\
\hline Facebook & $\begin{array}{l}\text { Старше } 25 \text { лет ( } 46 \% \\
\text { пользователей старше } \\
45 \text { лет) }\end{array}$ & 9 мин. & 56,2 млрд. & 1,3 млн. \\
\hline YouTube & $\begin{array}{l}\text { Старше } 12 \text { лет ( } 48 \% \\
\text { пользователей от } 25 \\
\text { до } 44 \text { лет) }\end{array}$ & - & 22 млрд. & 7,7 млн. \\
\hline Twitter & $\begin{array}{l}\text { До } 44 \text { лет (42\% поль- } \\
\text { зователей от } 25 \text { до } 34 \\
\text { лет) }\end{array}$ & - & 32,5 млрд. & 0,7 млн. \\
\hline
\end{tabular}

система «Яндекс» фокусируется на количестве человек в группе продвигаемого сайта.

К примеру, в случае, если в группе в социальной сети состоит более 500 пользователей, то поисковая система берет в расчет этот показатель при выстраивании ранжирования.

Социальные сети открывают значительные возможности для брендов, выстроивших свой рекламный поток, который создают сами потребители. Главным образом социальные сети обладают властью горизонтальных связей, поскольку пользователи склоны более доверять тем, кого они знают лично, в отличие от остальных типов рекламы. Маркетинг компании значительно ускорится, если рекомендации в социальных сетях разрастутся до уровня так называемого «сарафанного радио» [4].

Следующим цифровым маркетинговым каналом являются сайты и блоги. Сайты представляют собой определенную интернет-платформу, которой присвоено доменное имя, содержащую различную информацию и материалы. Значимая роль в конкурентном противостоянии за привлечение внимания потребителей отведена корпоративным сайтам.

Блоги же представляют собой определенную разновидность сайта, который содержит определенные сведения - видео, фото и текстовые материалы, которые пользователи добавляют по собственному желанию. В данной разновид- ности сайта пользователи рассказывают о своих целях, жизненных предпочтениях, наиболее значимой для него информации. Блог предполагает, таким образом, более неформальный стиль изложения. Он направлен на консолидацию пользователей по общим взглядам и интересам.

Сайты представляют собой своего рода окно в клиентскую среду - они способствуют усилению впечатлений - позитивных или негативных, в зависимости таких показателей, как доступность, легкость навигации и восприятия контента сайта. Сайт должен быть точкой, в которую собираются клиенты из общей цифровой среды, поэтому ключевым аспектом здесь является направление трафика на бизнес-сайт, который следует рассматривать, как центральный узел для бизнеса и его маркетинговых каналов, как цифровых, так и всех остальных.

Отдельное внимание следует уделить такие цифровым маркетинговым каналам, как «месендждеры». Данные мобильные приложения предназначены для обмена сообщения между пользователями. Программы данного типа пользуются высоким спросом у потребителей, поскольку месенджеры позволяют передавать необходимую информацию в текстовом, аудио или видео формате в кратчайшие сроки. На рис. 3 приведен индекс использования месенджеров в России в 2020 году. 


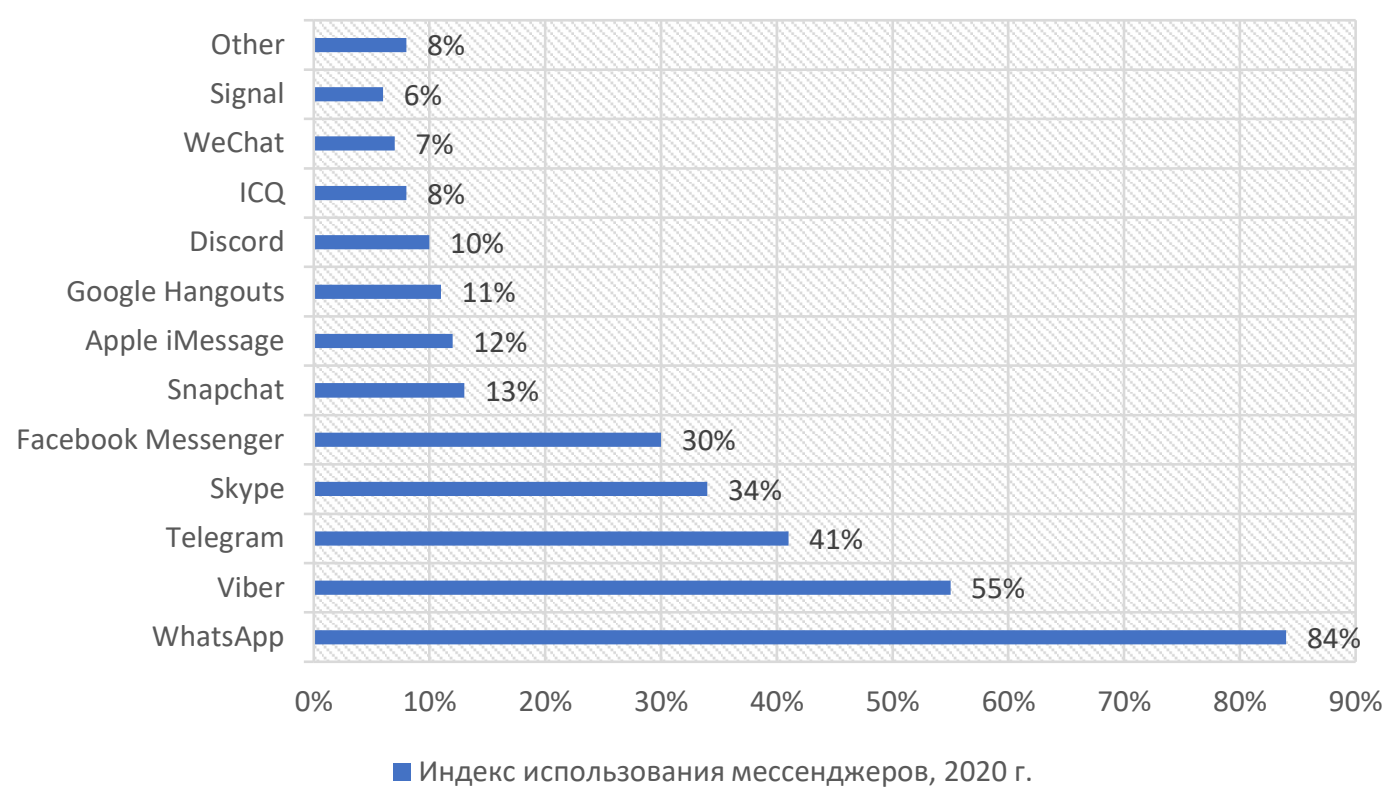

Рuc. 3. Индекс использования мессенджеров в России в 2020 году [8]

Ведущим мировым статистическим агентством «Statista» было проведено исследование использования потребителями в России мессенджеров. Для этого 1600 респондентам было предложено пройти онлайн-опрос, целью которого являлось определение индекса использования мессенджеров. Индекс использования мессенджеров представляет собой разность между процентом тех, кто установил мессенджер и не использует его и теми, кто установил мессенджер и пользуется им.

Таким образом, 84\% респондентов отметили, что часто используют мессенджер «WhatsApp», лишь 55\% тех, кто установил мессенджер «Viber» используют его, и 41\% мессенджер «Telegram» соответственно. Следовательно, ведущими мессенджерами в России являются «WhatsApp» и «Viber». Как правило, они применяются компаниями для связи с потребителями посредством текстовых сообщений с перечнем определенных торговых предложений. Согласно мнению Д.А.Шевченко «Мессенджер «WhatsApp» более востребован у интернет-магазинов, в то время как мессенджеры «Viber» выступает в качестве средства коммуникации между заказчиками и исполнителями различных видов услуг».

Таким образом, в рамках сложившейся экономической модели, для которой характерно применение цифровых технологий хозяйствующими субъектами при осуществлении своей деятельности в целях поддержания своих позиций на рынке и удовлетворения ожиданий потребителя возрастает и значительно расширяется роль цифровых маркетинговых каналов. Они выступают в роли цифрового пространства, которое соединяет между собой конечных потребителей с компаниями. Цифровой маркетинговый канал представляет собой место реализации не только функций продаж, но и обслуживания, коммуникаций. Он становится своего рода инструментом, который позволяет компаниям выстраивать долгосрочные взаимоотношения со своими клиентами, направленные на создание уникального, ценностного предложения потребителям.

\section{Библиографический список}

1. Шевченко Д.А. Цифровой маркетинг: обзор каналов и инструментов // Практический маркетинг. 2019. № 10 (272). URL: https://cyberleninka.ru/article/n/tsifrovoy-marketing-obzor-kanalov-i-instrumentov (дата обращения: 03.11.2021).

2. Шевченко Д.А. Основы современного маркетинга. / Д.А.Шевченко. - М.: Издательско-торговая корпорация «Дашков и К», 2019-41 С. 
3. С. Холл. В2В продажи: как построить эффективную систему продвижения / [переведено с английского]. Москва: Эксмо, 2020. - 448 с. - (Лучший мировой опыт)

4. С. Кингснорт. Стратегия цифрового маркетинга: интегрированный подход к онлайн-маркетингу / [пер. с англ. А. Огнёва]. - Москва: Олимп-Бизнес, 2019. - 416 с.: ил. ISBN 978-5-6040010-2-8

5. Лужнова Н. В., Усанова Е. А. Роль социальных сетей в интернет-маркетинге //Экономика и бизнес: теория и практика. - 2020. - № . 3-1.

6. Данько Т. П., Китова О.В. Вопросы развития цифрового маркетинга // ПСЭ. 2013. № 3 (47). URL: https:// cyberleninka.ru/article/n/voprosy-razvitiya-tsifrovogo-marketinga (дата обращения: 03.11.2021).

7. WEB-Index. Media Scope. Общая аудитория интернета. [Электронный ресурс] // URL: https://webindex. mediascope.net/general-audience (дата обращения: 31.10.2021)

8. Statista. Usage index of social media messengers in Russia in 2020. [Электронный ресурс] // URL: https://www. statista.com/statistics/1110818/russia-most-popular-messengers-on-social-media/

9. Statista. Number of smartphone users in Russia from 2015 to 2025. [Электронный ресурс] // URL: https://www. statista.com/statistics/467166/forecast-of-smartphone-users-in-russia/

10. Similarweb. Official Measure of the Digital World. [Электронный pecypc] // URL: https://www.similarweb.com/ (дата обращения: 01.11.2021)

11. Purwanti Y. et al. The Influence Of Digital Marketing \& Innovasion On The School Performance //Turkish Journal of Computer and Mathematics Education (TURCOMAT). - 2021. - T. 12. - № . 7. - C. 118-127.

12. Media Scope. Аудитория интернета в России в 2020 году. [Электронный ресурс] // URL: https://mediascope. net/news/1250827/ (дата обращения: 03.11.2021)

13. Desai V. Digital marketing: A review //International Journal of Trend in Scientific Research and Development. 2019. - T. 5. - № . 5. - C. 196-200.

14. Columbus, L. Mobile technologies becoming a growth engine for small and medium businesses, Forbes. [Электронный ресурс] // URL: https://www.forbes.com/sites/louiscolumbus/2015/02/08/mobile-technologies-becoming-agrowth-engine-for-small-and-medium-businesses/?sh=5972ca8e74ed/ (дата обращения 30.10.2021) 